\title{
Seis Sigma en Instituciones de Educación Superior en México
}

\author{
Carlos Gastelum-Acosta, Jorge Limon-Romero*, Marco Maciel-Monteon y Yolanda Baez-Lopez \\ Universidad Autónoma del Estado de Baja California, Facultad de Ingeniería, Arquitectura y Diseño, \\ Carretera Transpeninsular Ensenada-Tijuana, número 3917 colonia Playitas, Ensenada, Baja California, \\ México. (e-mail: jorge.limon@uabc.edu.mx).
}

*Autor a quien debe ser dirigida la correspondencia

Recibido Mar. 12, 2018; Aceptado May. 18, 2018; Versión final Jun. 20, 2018, Publicado Oct. 2018

\begin{abstract}
Resumen
El presente trabajo analiza los proyectos de mejora que se desarrollan en las Instituciones de Educación Superior en México, para evaluar la posible incorporación de la metodología Seis Sigma como una estructura para la realización de dichos proyectos. Para esto se aplicó una encuesta en la región noroeste de México y la información obtenida sugiere que la mayoría de las universidades basan sus esfuerzos en la norma ISO 9001, en los sistemas de acreditación externos y en menor medida utilizan otras estrategias. Sin embargo, en el desarrollo de estos proyectos se utilizan algunas herramientas fundamentales de Seis Sigma. También se observa que se cumplen algunos factores básicos que facilitan su implementación. Esto hace suponer que la integración de la estructura de la metodología DMAMC (Definir, Medir, Analizar, Mejorar y Controlar) a las estrategias de mejora que ya se utilizan es factible. Esto podría impactar positivamente en los procesos de las Instituciones de Educación Superior.
\end{abstract}

Palabras clave: educación superior; proyectos de mejora; seis sigma; beneficios; encuesta

\section{Six Sigma in Higher Education Institutions in Mexico}

\begin{abstract}
The present work shows an analysis of the way in which improvement projects are carried out in Higher Education Institutions in Mexico, to evaluate the possibility of incorporating the Six Sigma methodology as a structure for the development of such projects. For this, a survey was applied in the northwest region of Mexico and the information obtained suggests that the vast majority of universities base their strategies in the ISO 9001 standard, in external accreditation systems and to a lesser extent the use other strategies. However, in the development of these projects some tools that form a fundamental part of the SS methodology are used. It was also observed the presence of some basic factors that facilitate the implementation of the strategy. This suggests that the integration of the structure of DMAIC methodology (Define, Measure, Analyze, Improve and Control) to the strategies of improvement that are already used is feasible. This could positively impact the Higher Education Institutions processes.
\end{abstract}

Keywords: higher education; improvement projects; six sigmas; benefits; survey 


\section{INTRODUCCIÓN}

La competencia mundial por el posicionamiento en los mercados afecta de forma considerable a las empresas de manufactura y de servicios, por lo que deben mejorar sus procesos productivos y administrativos si quieren permanecer en esta dinámica por un largo periodo de tiempo. Esto desencadena un gran número de exigencias para todos los entes relacionados con dichas organizaciones; las Instituciones de Educación Superior (IES) no están exentas de estas exigencias ya que son generadoras de profesionales con las competencias demandadas por el entorno globalizado actual, para cubrir áreas especializadas de manufactura y de servicios.

Las IES en México, además de las exigencias del sector productivo y social en cuanto a la pertinencia de la oferta educativa, se enfrentan a las restricciones económicas del país, que involucran costos cada vez más elevados y los presupuestos asignados por los organismos de los gobiernos estatales y federales que apenas son suficientes para cubrir las necesidades del gasto operativo anual (Velázquez, 2014). Además, en el interior de las IES los estudiantes, padres de familia y empleadores consideran la calidad de la enseñanza como un referente importante de competitividad en el mercado laboral; por lo que se hace necesario la incorporación de herramientas para mejorar la calidad de los procesos de enseñanza, investigación, servicios, administrativos y de apoyo (Ramasubramanian, 2012). El desafío de las IES es dirigir esfuerzos para fortalecer sus estructuras administrativas con sistemas de aseguramiento de la calidad, con el objetivo de ser productivos y eficientes en el aprovechamiento de los recursos limitados y así lograr reducir el desperdicio para reasignar recursos sobre las áreas prioritarias que determinen las IES en sus planes de desarrollo (Velázquez, 2014). Para complicar aún más el ambiente de calidad en la educación, las técnicas de calidad de la industria generalmente se enfocan en los requisitos del cliente; sin embargo, la configuración de la educación superior hace que enfocarse en el cliente sea difícil. Mientras que los estudiantes de una institución de educación superior son quizás el cliente más obvio, muchos otros interesados también funcionan como clientes para las diferentes áreas de operaciones como lo son los padres de familia, profesores, gobierno, sociedad, futuros empleadores, colegios de graduados y casas certificadoras (Quinn et al., 2009).

Debido a esto, en la actualidad, algunas universidades alrededor del mundo han incorporado exitosamente estrategias de calidad para mejorar sus procesos, tales como, la Gestión Total de la Calidad (TQM por sus siglas en inglés), ISO 9001 y la metodología Seis Sigma (SS) (Pandi et al., 2016). De manera particular algunas universidades han aplicado la metodología SS como estrategia de mejora de la calidad en sus procesos, por ejemplo, en Arabia Saudita la Universidad Rey Abdullah de Ciencia y Tecnología (Antony y Cudney, 2016), en los Estados Unidos de América la Universidad de Buffalo (Jankowski, 2013), la Universidad Estatal de Valdosta y el Colegio Estatal Gordon (Sunder, 2016a), en el Reino Unido la Universidad de Newcastle (Kumi y Morrow, 2006), en Corea del Sur la Universidad de Sungkyunkwan (Kim, 2010), en Irán el Centro Regional de Información para Ciencia y Tecnología (Biranvand y Akbar, 2013), por mencionar algunas.

Con respecto a México, la literatura sobre proyectos de mejora (PM) y SS en IES es escasa, lo que plantea la necesidad de analizar las estrategias de mejora de procesos que siguen actualmente estas instituciones. Adicionalmente, no se ha encontrado evidencia previa de estudios sobre la factibilidad de implementar SS en PM en el contexto de las IES mexicanas. Por lo anterior, el objetivo del presente trabajo es generar información que ayude a determinar si las IES podrían integrar la metodología SS a los sistemas de gestión de la calidad que actualmente utilizan. Para lograr dicho objetivo es necesario determinar en una primera instancia cuales son las estrategias de mejora aplicadas en las IES en México y posteriormente cuales de los factores críticos para la implementación de SS son considerados desde el punto de vista del personal involucrado en la realización de los PM, a través de un cuestionario elaborado para este fin.

\section{El sistema de educación superior en México}

El sistema de educación superior en México está compuesto por IES particulares y públicas, las particulares conformadas por universidades, centros de estudios superiores, universidades tecnológicas e institutos tecnológicos de acuerdo a la Federación de Instituciones Mexicanas Particulares de Educación Superior (FIMPES); las públicas se integran por instituciones federales, estatales y de apoyo solidario; institutos tecnológicos; universidades politécnicas e interculturales; centros públicos de investigación; escuelas normales y centros de formación especializada (SEP, 2017).

La educación superior en México comprende los niveles de técnico superior universitario, con el objetivo de formar durante un periodo de dos o tres años en promedio a profesionales técnicos capacitados en un área específica; el nivel de licenciatura dividido en normal, universitaria y tecnológica. La licenciatura es impartida en periodos de cuatro o cinco años en promedio para formar profesionistas en las diferentes áreas del conocimiento en instituciones universitarias, tecnológicas y de formación de maestros. El nivel de posgrado sigue a la licenciatura y abarca especialidad, maestría y doctorado, siendo el objetivo la especialización en 
las diversas áreas del conocimiento. Durante el ciclo escolar 2016-2017 ingresaron a la educación superior un total de 3,762,679 estudiantes, cubriendo el 10.3\% de la matrícula total que incluye la educación básica y media superior. La matrícula de educación superior se distribuye en sus niveles con el $2.5 \%$ licenciatura normal, 91.2\% licenciatura universitaria y tecnológica y el 6.3\% para posgrado (SEP, 2017).

\section{Seis Sigma (SS)}

A principios de 1980 los productos japoneses tenían menor precio y mayor calidad por lo que eran más atractivos para el consumidor global. En respuesta a la amenaza japonesa para la fabricación estadounidense, varias iniciativas de calidad se introdujeron para ayudar a que la producción nacional de bienes y servicios fuera más competitiva. Motorola se enfrentaba a estos problemas como las demás industrias en esos momentos, entonces identificaron que estaban perdiendo gran parte de su empresa y productividad por los costos de la no calidad. Fue cuando el presidente de esta compañía en esa época, Bob Galvin, decidió que era necesario un esfuerzo mucho más intenso para resolver sus problemas. Un ingeniero de Motorola, Bill Smith, encontró que el nivel de calidad asociado con una medida de SS corresponde a una tasa de fracaso de dos partes por billón y lo adoptó como un estándar. El programa para lograr esta notable meta fue desarrollado en Motorola y llamado SS, que incluía muchas de las herramientas sistemáticas y rigurosas asociadas con los programas SS de hoy (Raisinghani et al., 2005).

Fue en 1988 cuando Motorola ganó el prestigioso Premio Nacional de Calidad Malcolm Baldrige debido en gran parte a su iniciativa llamada Seis Sigma (Gowen et al., 2008), así también por los resultados que presentaron: aumento en los niveles de calidad de varios productos y ahorros significativos de dinero. Para el año 2000, muchas de las principales corporaciones del mundo tenían una iniciativa SS en marcha, y en 2003 , se habían contabilizado más de 100,000 millones de dólares en ahorros combinados, por lo que SS se convirtió en el estándar mundial de la práctica empresarial de calidad (Reosekar y Pohekar, 2014).

Hoy en día la calidad en la educación se ha convertido en un concepto importante y varios autores han empezado a afirmar que la metodología surgida en las filas de Motorola puede convertirse en una necesidad para las universidades que quieran mejorar continuamente y así llegar a una excelencia de calidad educativa (Ho et al., 2006; Sunder, 2016b). Ésta metodología fomenta a que la toma de decisiones se basen en el análisis de datos y no solo en intuiciones o presentimientos (Báez et al., 2010), algo que sucede con frecuencia en las IES. El término SS empieza a sonar fuerte en el ambiente educativo, en especial por la creciente importancia de lo que llaman "calidad en la educación", particularmente en la educación superior, donde los productos finales del sistema pueden tener un impacto directo en la calidad de las organizaciones de los empleadores. SS se considera una estrategia viable, ya que es una metodología específica, mensurable y bien definida, se debe apreciar la utilidad, aplicabilidad e integración de las herramientas y técnicas SS para abordar problemas del mundo real (Ho et al., 2006).

\section{Aplicaciones exitosas de Seis Sigma en la educación}

En esta era de competencia cada vez mayor, SS puede ser una estrategia que apoye a las IES en su búsqueda de la mejorara continua de sus procesos. Como prueba del avance que se está dando respecto a la utilización de SS en las universidades a continuación se presentan algunos casos de aplicación de esta metodología y se enlistan los beneficios que se obtuvieron en cada caso de estudio.

De acuerdo a Kumi y Morrow (2006) se realizó una aplicación de SS en la biblioteca de la Universidad de Newcastle en Reino Unido. El proyecto se centró en mejorar el uso y la confiabilidad de la auto entrega que presta la institución en la biblioteca. Los autores documentan que el hacerlo bajo el esquema que proporciona SS les ayudó a centrar la atención en lo que el problema era en lugar de suposiciones sobre lo que el problema podría ser. De esta manera, el tiempo y los recursos estaban destinados a corregir lo que realmente estaba equivocado en lugar de ser utilizados al azar. Respecto a los beneficios obtenidos con este proyecto se logró lo siguiente: 1) reducir el número de empleados en el mostrador de entregas; 2) mover las unidades de auto entrega a ubicaciones más apropiadas; 3) aumentar el número de usuarios que podrían utilizar la auto entrega; 4) ampliación de los periodos de entrenamiento de los usuarios durante todo el año para que los beneficiarios utilicen el sistema; y 5) aumentar el uso del material disponible en biblioteca.

El siguiente caso de aplicación se desarrolló en la Universidad de Arizona donde se mejoró el préstamo entre bibliotecas a través de la aplicación de un enfoque de la metodología DMAMC. Según lo reportado por Voyles et al. (2008) a medida que las bibliotecas continúan evaluando las necesidades de servicio de sus clientes, es fundamental que consideren cómo se puede desarrollar el acceso y la prestación de servicios para apoyar las necesidades cambiantes de los clientes. Un análisis basado en datos de los procesos actuales, identificación de desfases en las expectativas de servicio y entrega real, un enfoque de equipo para descubrir y diseñar mejoras de procesos pueden asegurar que las bibliotecas sepan lo que están haciendo. Los 
beneficios obtenidos al llevar a cabo el proyecto para mejorar el proceso de préstamo de artículos diarios interbibliotecario, que incluye la obtención de retroalimentación de los clientes y la aplicación de soluciones, son los siguientes: 1) cambios en la plantilla para el turno vespertino y fines de semana sin aumentar el personal de préstamo interbibliotecario; 2) implementación de soluciones tecnológicas y compartir los cambios necesarios en el nivel de los consorcios para mejorar la tasa de cumplimiento y el tiempo de respuesta para el préstamo interbibliotecario; 3) cien por ciento de los artículos de préstamo interbibliotecario se entregan ahora electrónicamente a los escritorios de los clientes; y 4) el tiempo de respuesta para entrega de artículos se redujo al $70 \%$ de las solicitudes de artículos llenadas y entregadas en tres días o menos.

Por último, se presenta un caso diferente reportado por Oko y Kang (2015) en una IES de Nigeria, donde se revela que el proceso de admisión es inadecuado, acompañado de muchos ingredientes de desperdicios en el proceso. Es por esta y otras razones que se decidió investigar este proceso con el fin de ofrecer soluciones mediante un método mejorado que elimine los desperdicios, creando valor para los clientes y satisfacción de estos. El enfoque propuesto fue el de la metodología Lean Seis Sigma (LSS), donde se siguió la estructura DMAMC, en general se definió el problema que ya se tenía identificado, obtuvieron datos a través de una encuesta en la población que sería afectada por el proyecto, así también se calculó el tiempo de trabajo entre dos unidades consecutivas a través del número de horas trabajadas diarias y el número de formas para inscripción que se realizaban por día. En la fase de analizar encontraron que los métodos de comunicación y entrega de información a las diversas oficinas fueron muy pobres, el estudio también demuestra que los solicitantes tardan más tiempo en completar sus formularios y presentarlos.

En definitiva, la opinión general es que el medio electrónico debe utilizarse para el procesamiento de las admisiones, la colocación de anuncios de admisión y la colocación de los resultados de admisión a los candidatos a fin de reducir la incidencia de desperdicios en el proceso, se propusieron alrededor de seis mejoras donde destacan que las aplicaciones deben de ser en línea y calendarizadas. Las mejoras que obtuvieron fueron las siguientes: 1) reducción del tiempo de ciclo en 36.4\%; 2) el tiempo de espera se redujo en un $11.3 \%$; 3) el tiempo de utilización se mejoró un 40\%; 4) el re-trabajo se eliminó completamente; 5) el tiempo ocioso se redujo a 0 ; y 6 ) la eficiencia del ciclo del proceso se mejoró un 33\%. Se concluye que el trabajo ha sido un desafío que da una idea de la viabilidad de las aplicaciones de LSS en las administraciones y la gestión de las instituciones educativas, después de la aplicación se realizó pruebas y muestran una alta mejora en los procesos.

\section{METODOLOGÍA}

Para identificar si la metodología SS puede integrarse a las estrategias de mejora utilizadas en las IES mexicanas, fue necesario obtener información relacionada con el conocimiento que se tiene sobre esta metodología, cuáles sistemas de calidad tienen implementados, cómo se realizan los PM, si los responsables de calidad utilizan las técnicas y herramientas que se necesitarían para adoptar SS, entre otras cuestiones. Esta información se recolectó con un cuestionario dirigido al personal que haya participado en PM. El estudio se realizó en la zona noroeste de México, división que por operatividad realiza la Asociación Nacional de Universidades e Instituciones de Educación Superior (ANUIES) en México; zona que abarca los estados de Baja California, Sonora, Sinaloa, Baja California Sur y Chihuahua; la Universidad Autónoma del Estado de Baja California (UABC) pertenece a esta zona geográfica, lo que facilitó la obtención de la información y visitas a las instituciones educativas. Las IES públicas son analizadas en el presente trabajo ya que atienden a poco más del 70\% de la matrícula nacional de educación superior (SEP, 2017).

La zona noroeste de México cuenta con 362 unidades académicas en las IES públicas de los diferentes sistemas, siendo así el objetivo a encuestar, por lo que, primeramente, se llevó a cabo una revisión bibliográfica para el diseño y evaluación del instrumento para la recolección de datos. El cuestionario se dividió en cuatro secciones, la primera se refiere a los datos generales de las IES, la segunda sección cuestiona el uso de herramientas de calidad en los PM, la tercera sección indaga el conocimiento de la metodología SS y la cuarta sección sobre los beneficios obtenidos al aplicar PM. Una vez diseñado el cuestionario, se utilizó una plataforma para enviarlos electrónicamente, para aumentar la tasa de respuesta fue necesario el contacto vía telefónica y visitar las IES en sus localidades. Después de recolectar la información se procedió el análisis para evaluar la posible adopción de la metodología SS en las IES.

\section{RESULTADOS}

Se envió el cuestionario a 362 IES, de las cuales se recibieron 215 respuestas y solamente 144 cuestionarios estuvieron completos, representando un $40 \%$ de respuesta; estos provienen de 39 universidades diferentes. La figura 1 muestra el porcentaje de respuestas por estado, siendo Sonora y Sinaloa los de mayor aportación, con un $36 \%$ y un $21 \%$ respectivamente. Por otra parte, la figura 2 indica que un $40 \%$ de las personas 
encuestadas son jefes o coordinadores de unidades académicas; un $24 \%$ son profesores y en menores porcentajes contestaron directivos, administradores y coordinadores de gestión de calidad.

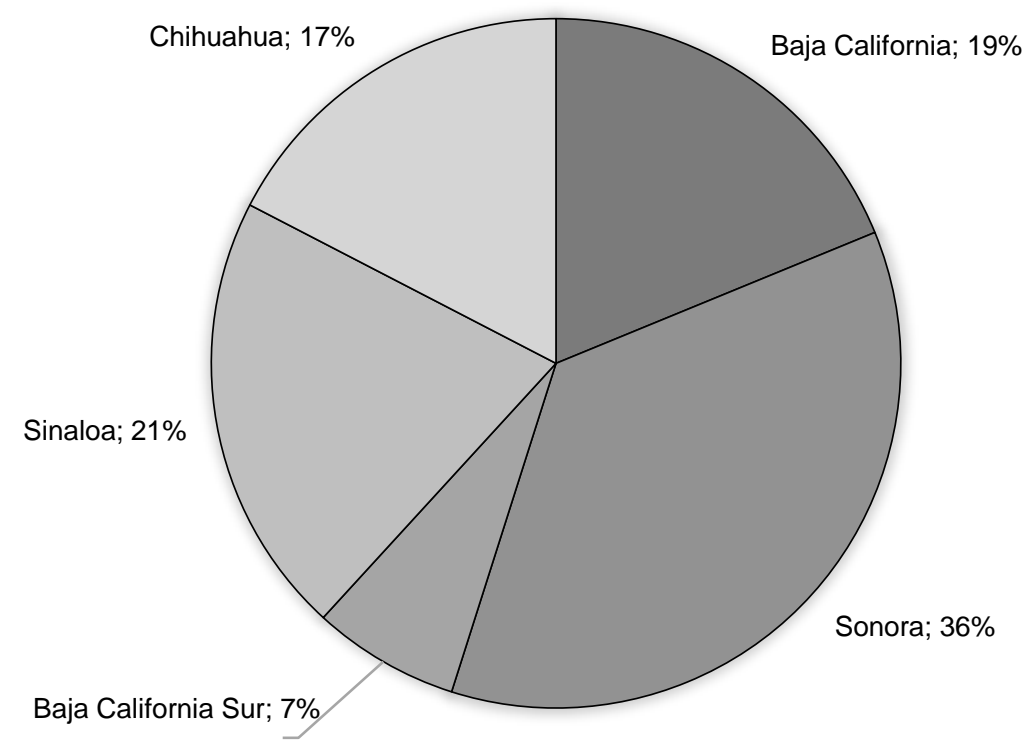

Fig. 1: Porcentaje de respuestas por estado

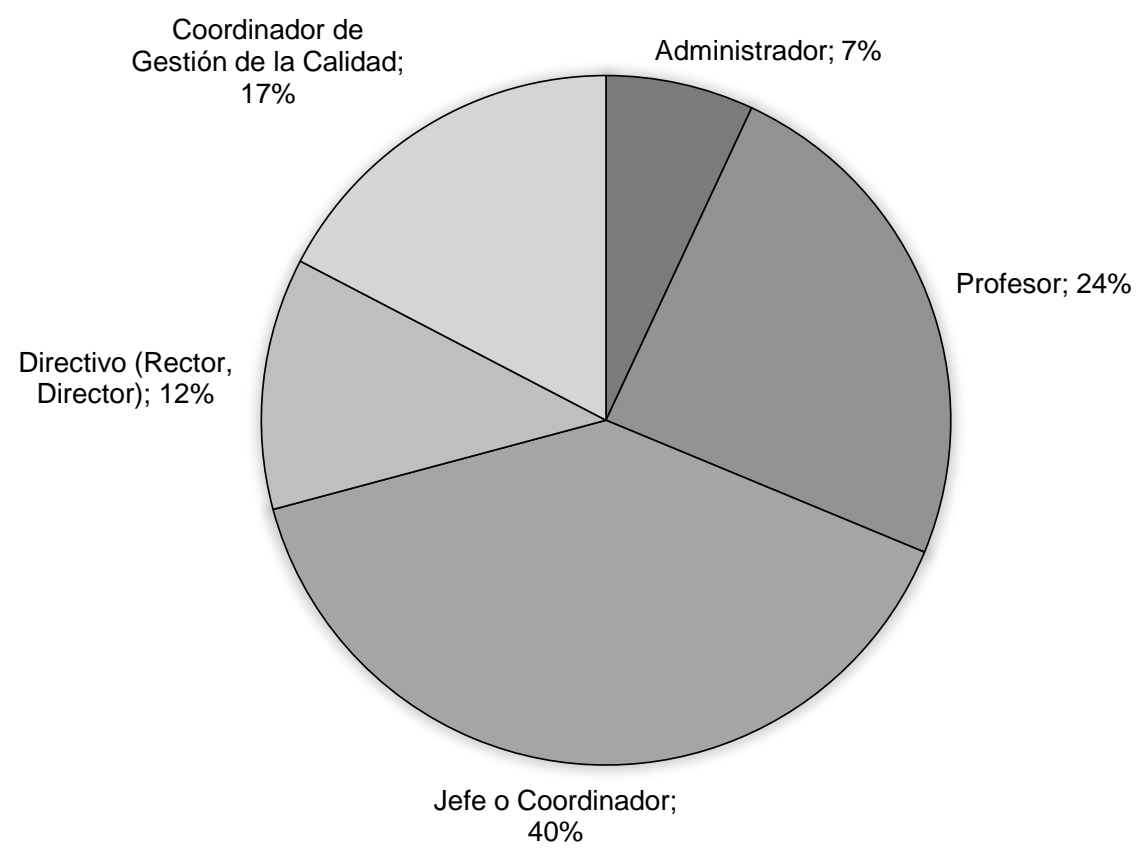

Fig. 2: Puestos de las personas encuestadas en porcentaje

Por otra parte, en la figura 3 se observa que las estrategias más conocidas para realizar PM son ISO 9001 con un $94 \%$, seguida de TQM y la metodología SS ambas con un $51 \%$, para finalizar con Manufactura Esbelta con el $40 \%$. En cuanto al nivel de uso de estas estrategias puede decirse que el $79 \%$ de los encuestados dice haber utilizado ISO 9001, 23\% utilizó TQM y solo el 6\% aplican la metodología SS, a pesar de que cerca del $50 \%$ la conocen.

En relación a las acreditaciones de los programas educativos, el $73 \%$ de los encuestados mencionó que la institución educativa en la que laboran cuenta con un departamento de gestión de la calidad, mejora continua o acreditaciones académicas. Con la información obtenida puede verse también que el $85 \%$ de las IES tiene programas educativos acreditados por organismos externos. De las asociaciones acreditadoras externas con mayores porcentajes de participación están el Consejo para la Acreditación de la Enseñanza de la Ingeniería 
A.C. (CACEI) ocupa el 66\%, el Consejo de Acreditación en Ciencias Administrativas Contables y Afines A.C. (CACECA) con $50 \%$ y los Comités Interinstitucionales para le Evaluación de la Educación Superior A.C. (CIEES) con un $40 \%$. En la actualidad, algunas IES han optado por utilizar organismos internacionales como la agencia acreditadora de ingeniería y tecnología en los Estados Unidos (ABET por sus siglas en inglés).

Uno de los elementos básicos para integrar SS a las estrategias de mejora de las IES es la utilización de herramientas y técnicas en las fases que componen la metodología DMAMC en la realización de PM, que es la parte medular de SS (Banuelas Coronado y Antony, 2002). En este sentido, la figura 4 muestra los porcentajes de utilización de las herramientas en la realización de los PM, destacando los diagramas de flujo con el $69 \%$, las hojas de verificación y el Análisis Causa- Efecto ambas con un $68 \%$, la técnica de tormenta de ideas con un $67 \%$, análisis de causa raíz con $51 \%$, el análisis de Pareto con un $45 \%$, histogramas con $42 \%$, y el mapeo de procesos con $39 \%$.

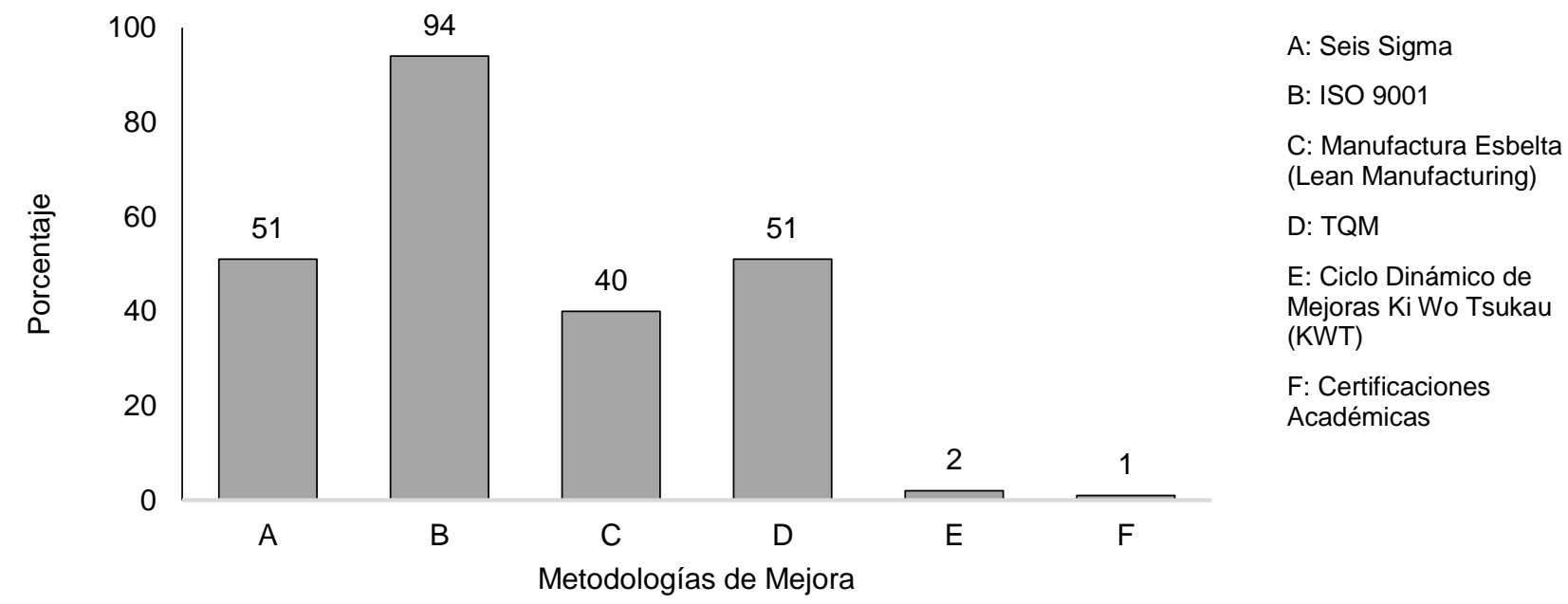

Fig. 3: Conocimiento de las estrategias de mejora en porcentaje

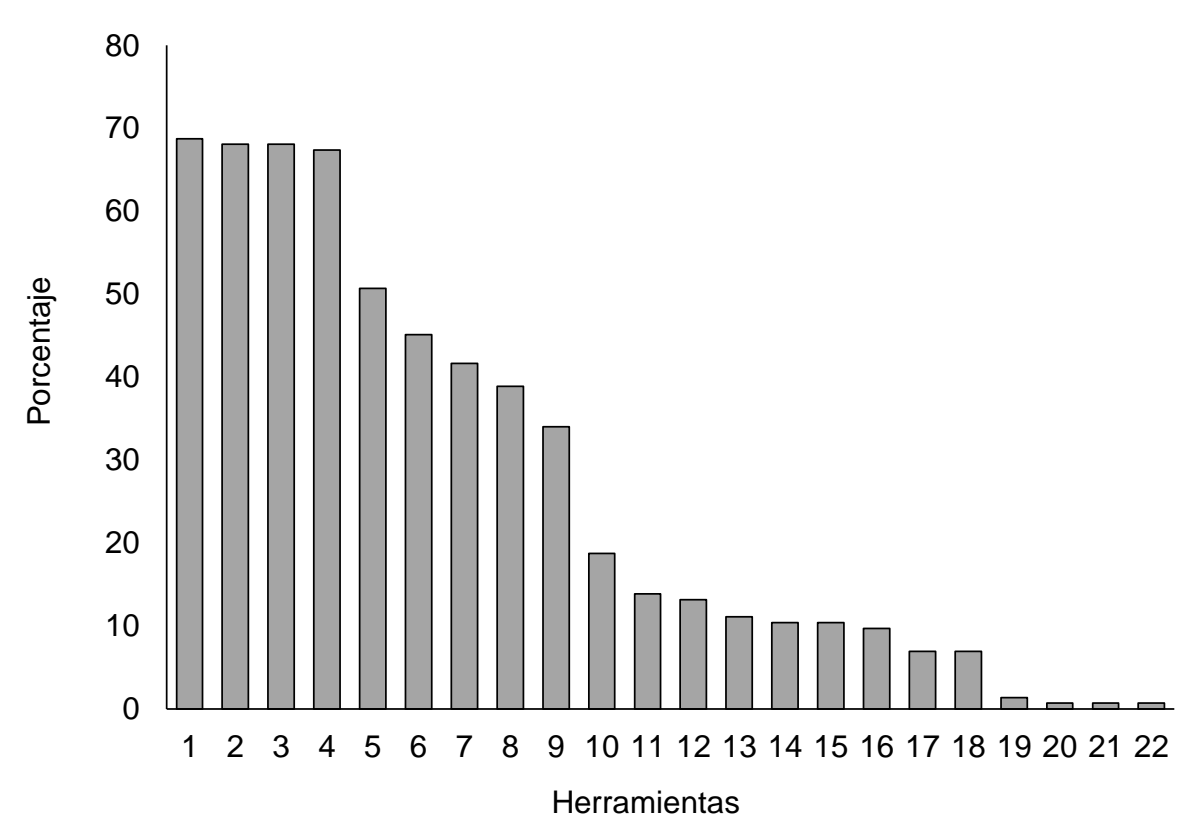

\author{
1: Diagrama de Flujo \\ 2: Análisis Causa Efecto \\ 3: Hoja de Verificación \\ 4: Tormenta de Ideas \\ 5: Análisis de Causa Raíz \\ 6: Análisis de Pareto \\ 7: Histogramas \\ 8: Mapeo de Procesos \\ 9: Gráficas de Control \\ 10: Plan de Control \\ 11: Análisis del Sistema de \\ Medición \\ 12: Análisis de Modo y Efecto de \\ la Falla(AMEF) \\ 13: Diagrama de Dispersión \\ 14: Análisis de Varianza \\ 15: Prueba de Hipótesis \\ 16: Análisis de Regresión \\ 17: Análisis de Capacidad de \\ Proceso \\ 18: SIPOC \\ 19: 5 Porqué \\ 20: Matriz de Marco Lógico \\ 21: Matriz FODA \\ 22: Ki Wo Tsukau, ciclo de \\ mejora continua
}

Fig. 4: Gráfica de herramientas utilizadas en porcentaje

Por último se presentan los beneficios obtenidos al realizar los PM, éstos se muestran en la tabla 1 , destacando que un $77 \%$ de los encuestados respondieron que casi siempre o siempre los PM facilitan el obtener o mantener las acreditaciones de los programas educativos, así también un $70 \%$ dijo que incrementa el nivel de calidad en el servicio y en los procesos, y con un $68 \%$ se mencionó como un beneficio obtenido la toma de decisiones basada en datos, mientras que solo un 51\% reportó haber obtenido beneficios financieros. 
Tabla 1: Beneficios reportados al aplicar PM

\begin{tabular}{lc}
\hline \multicolumn{1}{c}{ Beneficios } & Porcentaje \\
\hline Facilita el obtener o mantener la acreditación de los programas educativos. & $77 \%$ \\
Incremento Nivel de calidad del servicio y en los procesos. & $70 \%$ \\
Toma de decisiones basadas en datos. & $68 \%$ \\
Incremento en la Satisfacción del cliente (Estudiantes, profesores, personal administrativo, padres, & $67 \%$ \\
empleadores, colegio de graduados, comunidad). & $66 \%$ \\
Mayor posicionamiento de la Unidad Académica (Con alumnos potenciales y empleadores). & $63 \%$ \\
Mejora la Infraestructura y Equipamiento. & $62 \%$ \\
Mejora en la tasa de titulación. & $61 \%$ \\
Incremento del trabajo en equipo. & $60 \%$ \\
Mejora en alguno de los índices del rendimiento escolar (Reprobación, Deserción y Eficiencia & $53 \%$ \\
Terminal). & $51 \%$ \\
Mejora en los resultados de exámenes integradores (EGEL o similares). & \\
Beneficios financieros & \\
\hline
\end{tabular}

\section{DISCUSIÓN}

Las IES en la zona noroeste de México para llevar a cabo los PM utilizan varias de las herramientas que son reportadas por diversos autores en proyectos exitosos aplicados en procesos de IES (Sunder, 2016b; Antony et al., 2012; Isa y Usmen, 2015), por lo que solo restaría capacitarse en cuanto a la metodología SS para sistematizar la forma en que se debe de trabajar en cada una de las etapas del DMAMC, así como las herramientas que pudieran utilizar en cada una de ellas. Con esto, se aseguraría la estandarización requerida para realizar un PM y aumentar los beneficios obtenidos.

Entre los componentes más importantes de SS están el aspecto estadístico y el sistema de cinturones (Belt System), los cuales estarían cubiertos parcialmente, ya que la mayoría de las IES cuentan en su planta docente con profesores con los conocimientos estadísticos suficientes y se imparten asignaturas relacionadas con probabilidad y estadística cubriendo temas de estadística inferencial entre otros. Adicionalmente en las IES que cuentan con programas de ingeniería, específicamente con el perfil de ingeniería industrial y algunas carreras administrativas se cubren tópicos relacionado con el control estadístico de procesos, diseño de experimentos, gestión de sistemas de calidad, administración de la calidad y manufactura esbelta, por lo que solo restaría formalizar su entrenamiento en la gestión de proyectos y la metodología DMAMC para estructurar y dar seguimiento a los PM, a través de diplomados y certificaciones en el sistema de cinturones. La Universidad Estatal de Arizona ha implementado una certificación en la metodología SS, tomada por los alumnos en forma paralela a los programas de maestría y doctorado ofreciendo un contenido temático relacionado con tópicos sobre diseño de experimentos, análisis de regresión, control estadístico de calidad y metodología SS (Montgomery et al., 2005).

La implementación exitosa de la metodología SS en las IES, así como en cualquier organización requiere algunos factores básicos como la presencia de un liderazgo bien definido, el compromiso para la gestión de recursos, la vinculación con la estrategia de la universidad, el enfoque en el cliente y la selección adecuada del personal, el soporte y compromiso de la alta dirección, una comunicación efectiva en todos los niveles, la preparación para adoptar una nueva metodología, una adecuada selección y priorización de proyectos, la motivación hacía el personal para mejorar su actitud y disminuir la resistencia al cambio, el entrenamiento y la clara definición de métricas de rendimiento (Antony et al., 2012; Antony, 2014; Holmes et al., 2015). Estos factores son esenciales para la implementación y sostenibilidad de la metodología SS y deben existir antes de iniciar cualquier estrategia de mejora y así tener mayor probabilidad de éxito.

De acuerdo con la información analizada de la encuesta aplicada y la experiencia de los autores, puede decirse que las IES mexicanas cumplen con la mayoría de estos factores básicos, como se muestra en la tabla 2, donde 10 de los factores tienen una mediana de 4 de la escala Likert utilizada (1:nunca, 2:casi nunca, 3:a veces, 4:casi siempre y 5:siempre), por lo que se consideran resultados favorables respecto a la práctica de estos, por ejemplo, el compromiso y liderazgo de la alta dirección se manifiestan en la preocupación y disposición para la mejora continua al aplicar estrategias como las normas ISO, también está presente la gestión de recursos, ya que la gran mayoría cuenta con programas educativos acreditados por organismos externos nacionales y recientemente algunas IES están buscando ser acreditadas por organismos internacionales. La adecuada selección y priorización de proyectos es una práctica común en las IES 
encuestadas, ya que muestran participación en ellos a través de estrategias de mejora y la aplicación de herramientas y técnicas para realizarlos.

Tabla 2: Estadístico descriptivo de los factores básicos de SS en las IES

\begin{tabular}{lc}
\hline \multicolumn{1}{c}{ Factor } & Mediana \\
\hline Cambio Cultural & 4 \\
Métricas de Rendimiento Claras & 4 \\
Participación y Compromiso de la Dirección & 4 \\
Vinculación con el Cliente & 4 \\
Vinculación con la Estrategia de la Institución & 4 \\
Priorización y Selección de Proyectos & 4 \\
Educación y Entrenamiento & 4 \\
Comunicación & 4 \\
Vinculación con Recursos Humanos & 4 \\
Selección de los Miembros del Equipo y Trabajo en Equipo & 4 \\
Vinculación con Proveedores & 3 \\
\hline
\end{tabular}

Por otra parte, la incorporación de la metodología SS como un marco para realizar los PM en las IES puede ser muy atractiva, ya que como previamente se menciona y es reportado en la literatura, los beneficios obtenidos por las IES al aplicar SS en los PM pueden ser bastantes, entre los que se pueden mencionar, la obtención de ahorros financieros, mejora de los procesos que impactan directamente en los clientes, aumento en la satisfacción del personal y la realización de un proceso rápido para la admisión de estudiantes calificados (Sunder, 2016b), mayor transparencia de los procesos, mayor moral para los miembros del personal de las facultades, mejor trabajo interdisciplinario y, por lo tanto, se mejora el trabajo en equipo y el compromiso de los miembros del personal, reducción de costos y tiempo, reducción de la duplicación de trabajo en muchos departamentos y mayor conocimiento de la metodología de excelencia de procesos para mejorar la eficiencia y la efectividad (Antony y Cudney, 2016). Además se obtiene un mejor análisis de los procesos y la mejora en los servicios universitarios, eliminación de las medidas y actividades sin valor añadido, seguimiento a las evaluaciones de clientes inconformes, mejorar las habilidades de gestión de proyectos, revisión de los criterios de selección de los proveedores y mejoramiento de los sistemas de control de calidad (Isa y Usmen, 2015), aumento del apoyo financiero, desarrollo del profesorado, mejora de la calidad de la enseñanza, aumento en las tasas de graduación e identificación de problemas reales (Cudney et al., 2014).

Por lo anterior SS se muestra claramente como una estrategia que puede ayudar a las IES a mejorar las actividades relacionadas con su quehacer lo que directa o indirectamente tendría un impacto sustantivo en la mejora de la calidad de los servicios que ofrece. La implementación de SS no significa que se deba dejar de trabajar bajo la estrategia que han decidido adoptar (la norma ISO 9001 en la mayoría de ellas), si no que este enfoque nuevo daría un soporte y complemento para que el impacto sea mayor en los beneficios que se obtengan para todas las personas involucradas en los procesos administrativos y educativos, como los estudiantes, docentes, personal administrativo y de servicios, padres de familias, empleadores y la sociedad en general, como lo propone Marques et al. (2013).

\section{CONCLUSIONES}

En la presente investigación, se ha presentado un análisis de la aplicación de los PM con la finalidad de proponer la integración de la metodología SS en las estrategias de mejora de las IES mexicanas. LoS resultados del estudio, muestran que la propuesta de un enfoque de SS para llevar a cabo los PM en IES es factible, ya que cumplen varios elementos básicos para implementar la metodología SS con éxito, aplican herramientas y técnicas en la realización de proyectos de mejora y se obtienen beneficios con su aplicación.

Con base en la información de las IES de la zona noroeste de México se puede decir que las IES mexicanas que decidan integrar SS a sus estrategias de mejora deberán dirigir sus esfuerzos hacia la vinculación con los recursos humanos, a la selección del personal adecuado para implementar la metodología SS y tener una buena relación con los proveedores, que son los factores básicos que faltarían para aumentar la probabilidad de éxito en su incorporación, según los resultados de la encuesta y lo recomendado por diversos autores que han investigado este tema. 


\section{AGRADECIMIENTOS}

Se agradece al Programa para el Desarrollo Profesional Docente, para el Tipo Superior (PRODEP) por el apoyo para llevar a cabo el Proyecto DSA/103.5/16/10204 en la Facultad de Ingeniería, Arquitectura y Diseño de la Universidad Autónoma del Estado de Baja California.

\section{REFERENCIAS}

Antony, J. y E.A. Cudney, Lean Six Sigma Journey in a UK Higher Education Institute: Challenges, Projects, and Key Lessons Learned, 2016 ASEE Annual Conference and Exposition, New Orleans, United States 26-29 de Junio (2016)

Antony, J., N. Krishan, D. Cullen y M. Kumar, Lean Six Sigma for higher education institutions (HEls) Challenges, barriers, success factors, tools/techniques, doi: 10.1108/17410401211277165, International Journal of Productivity and Performance Management, 61(8), 940-948 (2012)

Antony, J., Readiness factors for the Lean Six Sigma journey in the higher education sector, doi: 10.1108/IJPPM-04-20130077, International Journal of Productivity and Performance Management, 63(2), 257-264 (2014)

Báez, Y.A., J. Limón, D.A. Tlapa y M.A. Rodríguez, Aplicación de Seis Sigma y los Métodos Taguchi para el Incremento de la Resistencia a la Prueba de Jalón de un Diodo Emisor de Luz, doi: 10.4067/S0718-07642010000100011, Información Tecnológica, 21(1), 63-76 (2010)

Banuelas-Coronado, R. y J. Antony, Critical success factors for the successful implementation of Six Sigma projects in organisations, doi: 10.1108/09544780210416702, The TQM magazine, 14(2), 92-99 (2002)

Biranvand, A. y K.A. Akbar, Evaluating the service quality in the Regional Information Center for Science and Technology using the Six Sigma methodology, doi: 10.1108/01435121311298270, Library Management, 34(1/2), 56-67 (2013)

Cudney, E.A., C.C. Elrod y S.M. Stanley, A systematic literature review of Six Sigma practices in education, doi: 10.1504/IJSSCA.2014.067552, International Journal of Six Sigma and Competitive Advantage, 8(3/4), 163-175 (2014)

Gowen, C.R., G.N. Stock y K.L. Mcfadden, Simultaneous implementation of Six Sigma and knowledge management in hospitals, doi: 10.1080/00207540802496162, International Journal of Production Research, 46(23), 6781-6795 (2008)

Ho, S., M. Xie y T. Goh, Adopting Six Sigma in higher education: some issues and challenges, doi: 10.1504/IJSSCA.2006.011564, International Journal of Six Sigma and Competitive Advantage, 2(4), 335-352 (2006)

Holmes, M.C., L.O. Jenicke y J.L. Hempel, A framework for Six Sigma project selection in higher educational institutions, using a weighted scorecard approach, doi: 10.1108/QAE-04-2014-0014, Quality Assurance in Education, 23(1), 30-46 (2015)

Isa, M.F.M. y M. Usmen, Improving university facilities services using Lean Six Sigma: a case study, doi: 10.1108/JFM-092013-0048, Journal of Facilities Management, 13(1), 70-84 (2015)

Jankowski, J., Successful Implementation of Six Sigma to Schedule Student Staffing for Circulation Service Desks, doi: 10.1080/15367967.2013.830930, Journal of Access Services, 10(4), 197-216 (2013)

Kim, D.S., Eliciting success factors of applying Six Sigma in an academic library, doi: 10.1108/14678041011026847, Performance Measurement and Metrics, 11(1), 25 - 38 (2010)

Kumi, S. y J. Morrow, Improving self service the six sigma way at Newcastle University Library, doi: 10.1108/00330330610669253, Program, 40(2), 123-136 (2006)

Marques, P., J. Requeijo, P. Saraiva y F. Frazao-Guerreiro, Integrating Six Sigma with ISO 9001 , doi: 10.1108/20401461311310508, International Journal of Lean Six Sigma, 4(1), 36-59 (2013)

Montgomery, D.C., R.K. Burdick y otros ocho autores, A University Six Sigma Based Program, doi: 10.1002/qre.631, Quality and Reliability Engineering International, 21, 243-248 (2005)

Oko, A. y P.S. Kang, Lean Six Sigma Approach to Improve the Admissions Process for a Nigerian HE Institute, International Journal of Scientific \& Engineering Research, 6(5), 368-378 (2015)

Pandi, A.P., P.R. Sethupathi y D. Jeyathilagar, Quality sustainability in engineering educational institutions: a theoretical model, doi: 10.1504/IJPQM.2016.076715, International Journal of Productivity and Quality Management, 18 (2 / 3), 364384 (2016)

Raisinghani, M.S., H. Ette, R. Pierce, G. Cannon y P. Daripaly, Six Sigma: concepts, tools, and applications, doi: 10.1108/02635570510592389, Industrial Management \& Data Systems, 105(4), $491-505$ (2005)

Ramasubramanian, P., Six Sigma in educational institutions, International Journal of Engineering Practical Research, 1(1), 1-5 (2012)

Reosekar, R.S. y S.D. Pohekar, Six Sigma methodology: a structured review, doi: 10.1108/IJLSS-12-2013-0059, International Journal of Lean Six Sigma, 5(4), 392-422 (2014)

SEP, Principales Cifras del Sistema Educativo Nacional 2016-2017, Dirección General de Planeación, Programación y Estadística Educativa, Ciudad de México, México (2017) 
Sunder, V., Constructs of quality in Higher Education services, doi: 10.1108/IJPPM-05-2015-0079, International Journal of Productivity and Performance Management, 65(8), 1091-1111 (2016a)

Sunder, V., Lean Six Sigma in higher education institutions, doi: 10.1108/IJQSS-04-2015-0043, International Journal of Quality and Service Sciences, 8(2), 159-178 (2016b)

Quinn, A., G. Lemay, P. Larsen y D.M. Johnson, Service quality in higher education, doi: 10.1080/14783360802622805, Total Quality Management, 20(2), 139-152 (2009)

Velázquez, C. J., Propuesta para aplicación de la metodología de seis sigmas en los procesos de la Universidad Estatal de Sonora, Global Conference on Business and Finance Proceedings, 9(1), 1271-1282, Honolulu, Hawai January 6-9 (2014)

Voyles, J. F., L. Dols y E. Knight, Interlibrary Loan Meets Six Sigma: The University of Arizona Library's Success Applying Process Improvement, doi: 10.1080/10723030802533911, Journal of Interlibrary Loan, Document Delivery \& Electronic Reserve, 19(1), 75-94 (2008) 